OPEN ACCESS

Edited by:

Ivana Dusan Pajic-Lijakovic,

University of Belgrade, Serbia

Reviewed by:

Sebastian Mueller,

Heidelberg University, Germany

Guozhu Ye

Institute of Urban Environment (CAS),

China

*Correspondence:

Jizeng Wang

jzwang@|zu.edu.cn

Specialty section:

This article was submitted to Biophysics,

a section of the journal

Frontiers in Physiology

Received: 22 March 2021

Accepted: 13 August 2021

Published: 01 September 2021

Citation:

Li R, Bu Y, Yang $C$ and Wang $J$ (2021) Effects of Lipid Deposition on Viscoelastic Response in Human

Hepatic Cell Line HepG2.

Front. Physiol. 12:684121.

doi: 10.3389/fphys.2021.684121

\section{Effects of Lipid Deposition on Viscoelastic Response in Human Hepatic Cell Line HepG2}

\author{
Rui Li, Yang Bu, Chendong Yang and Jizeng Wang*
}

Key Laboratory of Mechanics on Disaster and Environment in Western China, Ministry of Education, College of Civil Engineering and Mechanics, Lanzhou University, Lanzhou, China

Hepatic steatosis is associated with various liver diseases. The main pathological feature of steatosis is the excessive lipid accumulation. Ultrasound has been extensively used for the diagnosis of hepatic steatosis. However, most ultrasound-based non-invasive methods are still not accurate enough for cases with light lipid infiltration. One important reason is that the extent to which lipid infiltration may affect mechanical properties of hepatocytes remains unknown. In this work, we used atomic force microscope and in vitro dose-dependent lipid deposition model to detect the quantitative changes of mechanical properties under different degrees of steatosis in a single-cell level. The results show that hepatic cells with lipid deposition can be treated as linear viscoelastic materials with the power law creep compliance and relaxation modulus. Further analysis showed that even slight accumulation of lipid can lead to measurable decrease of stiffness and increased fluidity in liver cells. The accurate detection of viscoelastic properties of hepatocytes and the analysis methods may provide novel insights into hepatic steatosis grading, especially in the very early stage with reversible liver lesion. The application of viscoelasticity index for grading fat deposition might be a new detection indicator in future clinical diagnosis.

Keywords: liver steatosis, viscoelasticity, atomic force microscopy, oleic acid, HepG2 cells

\section{INTRODUCTION}

Hepatic steatosis is associated with various liver diseases (Sanyal et al., 2010; Hoyles et al., 2018). Some major diseases associated with steatosis include non-alcoholic fatty liver disease (NAFLD) and alcoholic liver disease (ALD) (Seitz et al., 2018; Gu et al., 2019), which are the major chronic liver diseases with a growing incidence every year. In severe cases, NAFLD can lead to liver dysfunction, hepatitis, and cirrhosis. An effective drug that can prevent and treat NAFLD has not been developed. Most patients with NAFLD may have no obvious clinical symptoms (Krawitz and Pyrsopoulos, 2020). Early control and intervention can effectively reverse disease progression. Thus, early diagnosis is highly important for patients with NAFLD.

Pathological diagnosis is the gold standard for confirming steatosis (Castera et al., 2019). This diagnosis procedure is invasive and is only used for the final determination for severe cases. However, early pathological changes usually occur in a certain part of the liver or dispersed in the liver. The recommended size for the biopsy is usually $1.5-3 \mathrm{~cm}$ (Rockey et al., 2009). The evaluation of focal lesions by invasive diagnosis is still affected by sampling error. Non-invasive imaging 
methods are widely used in clinical practice as alternative to liver biopsy (Lee and Park, 2014), and these methods include ultrasound (US), computed tomography, and magnetic resonance (MR) (Saadeh et al., 2002; Lee et al., 2010; Lee and Park, 2014; Zhang et al., 2014; Petäjä and Yki-Järvinen, 2016). The US diagnosis of hepatic steatosis is the most common imaging method, because it its widely used, safe, and inexpensive (Mazhar et al., 2009). However, US only has a reliable assessment in moderate and severe degree of steatosis $(\geq 30 \%)$ but is not accurate for steatosis; it only meets the diagnostic criteria or is within mild degree ( $<20-30 \%$; Dasarathy et al., 2009; Hernaez et al., 2011; Lee and Park, 2014). Therefore, high accuracy is still highly required in the US.

The inaccurate quantification of mild and local steatosis is related to the unclear changes in the mechanical properties of diseased liver tissue and the resolution of image feature recognition. The principle of US imaging method is based on the mechanical wave propagation in elastic media. Its propagation speed and amplitude are affected by the elastic modulus and bulk modulus of the medium. For viscoelastic (incomplete elasticity) media, the mechanical wave attenuates inside, and the attenuation speed is positively related to the viscosity of the medium (Righetti et al., 2002, 2003; Mueller, 2020b). Therefore, the US is an imaging method that is based on the differences in tissue mechanical properties. However, the imaging resolution is affected by input factors, such as wavelength, frequency, and wave constraint width. At present, the resolution of clinical device can only reach the millimeter level. Mild degree steatosis is limited to the lipid deposition in scattered cells, and reaching the resolution of the deposition area is difficult to reach, making US ineffective for the diagnosis of mild cases. The main pathology feature of liver steatosis is the excess lipid accumulation in liver. In mild cases, only local or small amount of lipid deposition is observed in hepatocytes. Therefore, the quantitative characterization of lipid deposition on the mechanical properties of liver tissue is needed for the accurate identification of mild fatty infiltration. How lipid deposition in liver cells affects the mechanical properties is essential for the accurate diagnosis of liver tissue lesions. A significant amount of work has been done in clinical practice through in vivo and ex vivo studies. Liver stiffness representing solid character has been studied as elastic moduli for years (Mueller and Sandrin, 2010). Yin et al. (2007) showed that steatosis has no effect on the stiffness of liver tissue via MRI measurements. They speculated that the stiffness range of fatty tissue is similar to the normal liver. Thus, the existence of hepatic steatosis in liver tissue has no obvious effect on the shear stiffness. A study measured by transient elastography on patients with ALD showed that steatosis is not related to liver stiffness (Rausch et al., 2016). Mueller (2020a) listed the stiffness of various living tissues quantified by elastography showed that the stiffness of fat tissue is about six times lower than that of the liver. Viscosity is another reference factor, suggesting that fat has an influence on mechanical properties. Barry et al. (2014) proved that fat adds viscosity to mouse livers and human liver samples. They suggested that the viscosity has a potential for steatosis scoring. Zhu et al. (2015) used gelatin-based phantoms containing a different ratio of castor oil to mimic different degrees of steatosis.
Their results showed a viscosity increment with the addition of castor oil. However, a prospective clinical study using shear wave elastography found that viscosity has a remarkable effect with fibrosis rather than steatosis (Deffieux et al., 2015). Moreover, fat is a soft material with fluidity that lowers mechanical properties in liver tissue (Karlas and Mueller, 2020), which may help attenuate the harmful effects of mechanical energy generated by impulse waves (Mueller, 2016). Whether steatosis affects the mechanical properties of liver remains to be determined (Mueller et al., 2020).

From a perspective of materials, liver tissue is a composite material containing hepatocyte, hepatic sinusoid, perisinusoidal cells, and tissue matrix. The overall mechanical properties are related to each component as described in the sinusoidal pressure hypothesis (Mueller, 2016). However, only the hepatocytes are mainly affected by fat deposition. Thus, the mechanical properties of single hepatocyte affected by different degrees of fat depositions should be studied to quantitatively describe the relationship between mechanical properties changes of hepatic lobule and fat deposition. Furthermore, a reference value for the diagnostic criteria of fatty liver in precise localization and classifications should be provided.

Many attempts have been focused on the use of atomic force microscopy (AFM) to characterize the mechanical fingerprint of healthy, fibrosis, and malignant liver cells (Braet et al., 2018). Hepatoma cells with different metastasis ability showed disparity in terms of the distribution patterns of Young's modulus (Tian et al., 2015). Differences in elastic modulus are found between normal liver, hepatoma, liver embryonic stem cells (Kim et al., 2013; Sun et al., 2016; Tsikritsis et al., 2016). An AFM topology study showed that the increased surface roughness and reduced cytoskeleton height are associated with curcumin-induced G2/M phase arrest in HepG2 cells (Jiang et al., 2013). These findings demonstrate the advantages of using AFM as a nanoscale measurement tool in capturing the biomechanical characteristics of liver cells. However, living cells are soft materials with rheological properties (Desprat et al., 2005). Hepatocytes are essential for whole-body energy metabolism and synthesis. The biosynthetic reaction is reflected by the composition change of cytoplasm rather than cytoskeletal structure, and this process might be difficult to capture based on transient elastic changes. Cytoplasmic component may not contribute to shear deformation of the cells, but it is highly incompressible. Considering that the cytoplasm is rich in different molecules such as lipids, carbohydrates, and proteins, dynamic changes in cell composition may be reflected in energy dissipation and time-dependent deformations. Our previous work has showed the viscoelastic differences between different cells in the liver ( $\mathrm{Bu}$ et al., 2019). Therefore, the viscoelastic parameters may be used as indicators to distinguish cells with different lipid depositions.

The human hepatoma cell line HepG2 preserves part of the hepatic cell characteristics, and it has been widely used to study hepatocyte functions (Knowles et al., 1980). In this work, an oleic acid (OA)-induced hepatocyte deposition model with a gradient change was used to simulate intracellular lipid deposition. Following our previous study (Bu et al., 2019), we adopted a 
three-parameter power-law-type constitutive relation to fit the experimental measurements, and this method is better than the five-parameter classical spring-dashpot. The combination of the proposed power law expression and AFM indentation measurements on the creep compliance and relaxation modulus will provide a unique way in determining the viscoelastic properties of the HepG2 cells with different lipid deposition levels. Based on this technique, we expect that the experimental results can quantitatively reveal the dependence of viscoelastic properties of cells on their lipid accumulation.

\section{MATERIALS AND METHODS}

\section{Cell Lines and Cell Culture}

Human hepatoma cell line HepG2 (Cell Bank of the Chinese Academy of Sciences, Shanghai, China) was cultured in Dulbecco's modified Eagle's medium (Gibco, Thermo Fisher Scientific, United States) containing 10\% (v/v) fetal bovine serum (Gibco ${ }^{\mathrm{TM}}$, Thermo Fisher Scientific, United States) supplemented with $100 \mathrm{U} / \mathrm{ml}$ penicillin and $100 \mu \mathrm{g} / \mathrm{ml}$ streptomycin in an incubator (INE800749L, Memmert, Germany) containing $5 \% \mathrm{CO}_{2}$ at $37^{\circ} \mathrm{C}$. Cells in logarithmic growth phase were used in the experiments.

\section{OA/BSA Complex Preparation}

The OA/BSA complex solution was prepared as described before (Cousin et al., 2001; Yun et al., 2006). Briefly, $100 \mathrm{mM}$ OA (Sigma-Aldrich, United States) solution was added to $10 \%$ fatty acid-free BSA (Solarbio, Beijing, China) stock solution. The mixture was incubated in a water bath for $30 \mathrm{~min}$ at $55^{\circ} \mathrm{C}$ to prepare a $5 \mathrm{mM} \mathrm{OA} / 10 \%$ BSA complex. The solution was filter-sterilized through a $0.22-\mu \mathrm{m}$ syringe filter (Millipore Corporation, Bedford, United States) after cooling to room temperature. The complex was used within 4 weeks.

\section{Proliferation Assay}

The proliferation rate was evaluated by 3-(4,5-dimethylthiazol2-yl)-2,5-diphenyltetrazolium bromide (MTT) assay (SigmaAldrich, MO, United States). HepG2 cells were seeded into a 96 -well plate $\left(3 \times 10^{3}\right.$ per well; Corning, NY, United States $)$ and cultured for $24 \mathrm{~h}$ at $37^{\circ} \mathrm{C}$. Then, DMEM containing different concentrations of FFA/BSA complex solution were added. After incubated for $24-72 \mathrm{~h}, 20 \mu \mathrm{L}$ of MTT $(5 \mathrm{mg} / \mathrm{mL})$ was added to each well and incubated at $37^{\circ} \mathrm{C}$ for $4 \mathrm{~h}$. The media was then removed, and $150 \mu \mathrm{L}$ of dimethyl sulfoxide (Sigma-Aldrich, MO, United States) was added to each well to dissolve the crystal. The absorbance was measured at a wavelength of $570 \mathrm{~nm}$ by using a microplate reader (Infinite 200 PRO, TECAN, Switzerland).

\section{Oil Red O (ORO) Staining}

Lipid droplets in cells were stained with ORO staining kit (Solarbio, Beijing, China). HepG2 cells were seeded on chamber slides. After treatment for $24 \mathrm{~h}$, cells were fixed with $4 \%$ buffered paraformaldehyde for $30 \mathrm{~min}$ and washed for three times with PBS. The slides were stained according to the manufacturer's instructions. Morphological changes were observed under light microscopy (Olympus, Tokyo, Japan). Positive area was measured using Image J software (Version 1.52, National Institutes of Health, United States). The extent of intracellular oil lipids was measured as described by Cui et al. (2010). ORO was extracted by isopropanol. The absorbance was measured at a wavelength of $405 \mathrm{~nm}$ by using a microplate reader (Infinite 200 PRO, TECAN, Switzerland).

\section{Immunofluorescent Staining}

HepG2 cells were seeded on chamber slides. After treatment for $24 \mathrm{~h}$, cells were fixed with $4 \%$ buffered paraformaldehyde for $30 \mathrm{~min}$ and washed thrice with PBS. For the detection of cell apoptosis, nuclei were labeled with Hoechst 33342 at $0.5 \mathrm{mg} / \mathrm{mL}$ for $15 \mathrm{~min}$ and washed with PBS. For actin cytoskeleton staining, cells were permeabilized with $0.1 \%$ Triton X-100 in PBS for 3 min and blocked with 1\% BSA. The slides were stained with FITC-labeled Phalloidin (Sigma-Aldrich, MO, United States) for $1 \mathrm{~h}$. Slides were sealed with ProLong glass antifade mountant (Thermo Fisher Scientific, MA, United States) and stored at room temperature for $24 \mathrm{~h}$ in the dark. The slides were observed under fluorescence microscope (Olympus, Tokyo, Japan). Fluorescence intensity was measured using Image J software (Version 1.52, National Institutes of Health, United States).

\section{AFM Indentation Assay}

The AFM indentation assay and the theoretical model were conducted as previously described (Bu et al., 2019). Cells were seeded and treated in sterilized $35-\mathrm{mm}$ petri dishes. A NanoWizard III AFM (JPK Instruments, Berlin, Germany) was used for the creep measurements of the cells. A silicon nitride AFM cantilever (NovaScan, Chicago, United States) with a polystyrene bead of $4.5 \mu \mathrm{m}$ diameter was used. The spring constant was $0.01 \mathrm{~N} / \mathrm{m}$. The cantilever was approached to the cell at a velocity of $50 \mu \mathrm{m} / \mathrm{s}$ until the preset force was reached. Once a preset force for the creep was reached, the approaching was stopped by controlling the position of cantilever base. The force was kept constant for $10 \mathrm{~s}$, and the cantilever retracted at a velocity of $1 \mu \mathrm{m} / \mathrm{s}$. Each cell was only approached once. At least 60 individual cells were tested in each group.

\section{Theoretical Model and Data Processing}

The hepatic cells are assumed as incompressible linear viscoelastic materials (Costa, 2003), and the stress-strain relationship can be defined through the creep compliance, $J(t)$, which is expressed as follows:

$$
\varepsilon_{i j}(t)=\int_{0}^{t} J(t-\tau) \frac{d \sigma_{i j}(\tau)}{d \tau} d \tau .
$$

Following Bu et al. (2019), we choose the power-law-type creep kernel function as follows:

$$
J(t)=\frac{1}{E_{0}}\left(\frac{t}{\tau_{0}}\right)^{\beta}
$$

where $E_{0}$ is the elastic modulus of viscoelastic material at time $\tau_{0}$, and $\beta$ characterizes the degree of dissipation or "fluidity" of the material. If $\beta$ approaches zero, then Equation 
(2) degrades into the Hook Law. If $\beta$ approaches unity, then Equation (2) just corresponds to the Newtown fluid. In the following sections, we set $\tau_{0}$ to a very small timescale $\left(\sim 10^{-5} s\right)$, then $E_{0}$ represents the instantaneous stiffness of the cell (Kollmannsberger and Fabry, 2011).

In AFM indentation tests, the tip of the AFM probe is considered as rigid sphere indenter, and the cells are treated as a viscoelastic half space. By neglecting the interfacial friction, the Hertz-Sneddon theory (Sneddon, 1965) is adopted to describe the indentation process. The relationship between the indentation depth, $\delta$, and indentation force, $P$, can be written as (Yang, 1966) follows:

$$
\delta^{3 / 2}(t)=\frac{3}{8 \sqrt{R}} \int_{0}^{t} J(t-\tau) \frac{d P(t)}{d \tau} d \tau
$$

where $R$ is the radius of polystyrene bead.

In our creep experiments, the indentation force can be pre-set as a step function as follows:

$$
P(t)=P_{0} \mathrm{H}(t)
$$

in which $P_{0}$ is the amplitude of the loading force, and $\mathrm{H}(t)$ is the so-called Heaviside step function. By submitting Equation (4) into Equation (3), the measured creep function can be obtained as follows:

$$
J(t)=\left\langle\frac{8 \sqrt{R}}{3 P_{0}} \delta^{3 / 2}(t)\right\rangle
$$

Notably, $J(t)$ in Equation (5) is obtained by performing ensemble average on sufficiently large number of independent experiments.

Figure 1 shows the process of a single-cell indentation. Contact point is defined as the moment when the indenter first contact with the surface of cells, where $Z$ is the vertical height of cantilever base, and $Z_{0}$ is the value of $Z$ just at the contact point, $d$ is the cantilever deflection at the location of tip, and $d_{0}$ is its value at contact point. Then, the indentation depth $\delta$ can be expressed as follows:

$$
\delta=Z-Z_{0}-\left(d-d_{0}\right)
$$

We assume that the cantilever is deformed like a Hookean spring with elastic constant, $k$. Then the indentation force and cantilever deflection can be related as follows:

$$
P=k\left(d-d_{0}\right) \text {. }
$$

where $d^{\prime}=P_{0} / k+d_{0}$. By keeping the indentation force constant, $P_{0}$, the creep compliance can be obtained as follows:

$$
J(t)=\left\langle\frac{8 \sqrt{R}}{3 P_{0}}\left[Z(t)-Z_{0}-d_{0}-d^{\prime}\right]^{3 / 2}\right\rangle
$$

\section{Statistical Analysis}

All statistical analyses were performed using SPSS 26 (IBM, NY, United States) and GraphPad Prism software 7.0 (GraphPad Software, CA, United States). Data were expressed as means \pm standard deviation. Statistical comparisons of the results were carried out using one-way ANOVA followed by the Bonferroni correction. $P$-value of $<0.05$ was considered statistically significant.

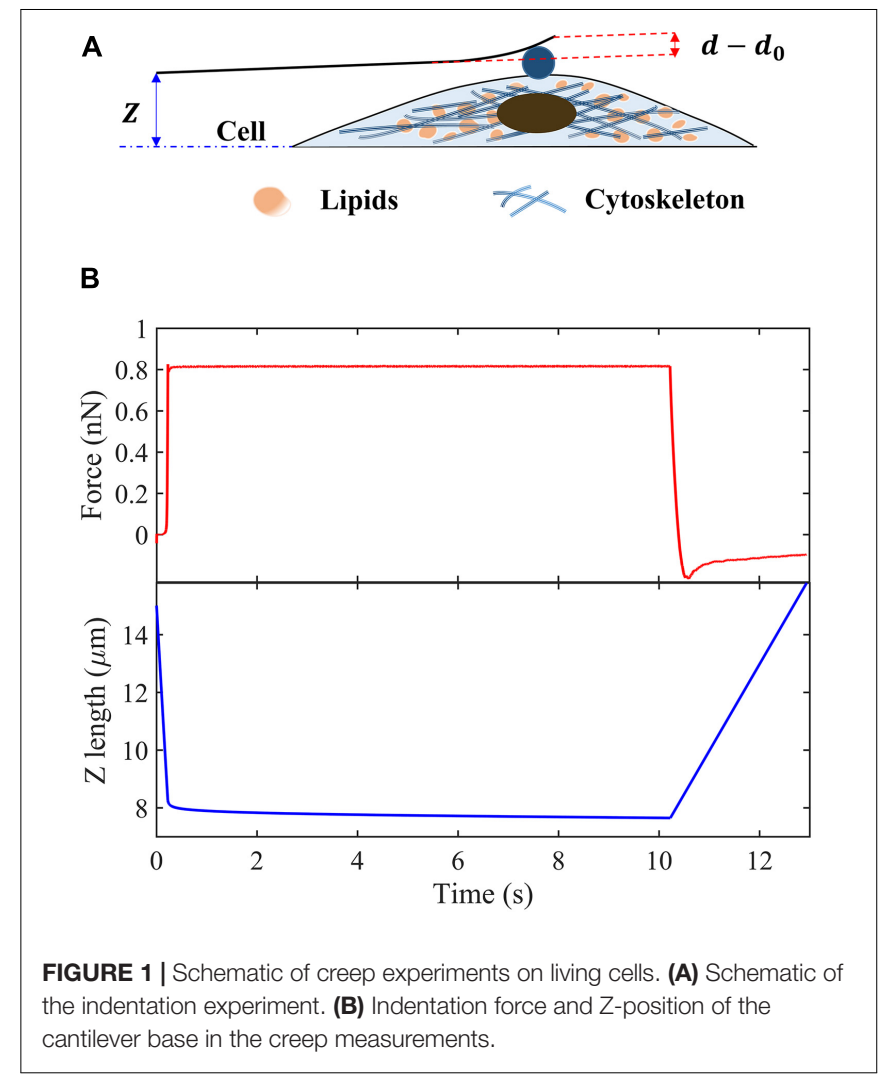

\section{RESULTS}

\section{Toxicity of OA on HepG2 Cells}

Cytotoxicity assay was carried out to determine the dosedependent response of OA on HepG2 cells. As shown in Figure 2A, HepG2 cells exposed to OA concentration lower than $1.5 \mathrm{mM}$ did not show proliferation inhibition after $24 \mathrm{~h}$. Cells exposed to 2 and $2.5 \mathrm{mM}$ reduced the viability rate at 24, 48, and 72 h significantly. Hoechst 33342 staining was used to detect apoptosis after $24 \mathrm{~h}$ (Figure 2B). Mitotic cells were incidentally detected. Cells exposed to OA concentration higher than $1.5 \mathrm{mM}$ showed increased chromatin condensation, nuclear fragmentation, and apoptotic bodies after $24 \mathrm{~h}$. The majority of cells nuclei morphology showed no apparent abnormality under $1.5 \mathrm{mM}$. Therefore, a concentration lower than $1.5 \mathrm{mM}$ and time of $24 \mathrm{~h}$ were chosen as the optimal conditions to observe the dose-dependent effect of HepG2 cell lipid deposit.

\section{OA Increased Lipid Deposition on HepG2 Cells}

We performed ORO staining to determine the intracellular lipid deposit for OA-induced lipid accumulation (Figure 3). Treatment with OA induced obvious fat deposition in HepG2 cells. The scattered lipid droplet increased the density and size with OA concentration. Considering the excessive deposition of lipids, lipid droplet fused into larger lipid vesicles, or the nuclei were pushed to one side (Figure 3A). The positive staining area of ORO staining showed an increase from $0.12 \% \pm 0.02 \%$ 


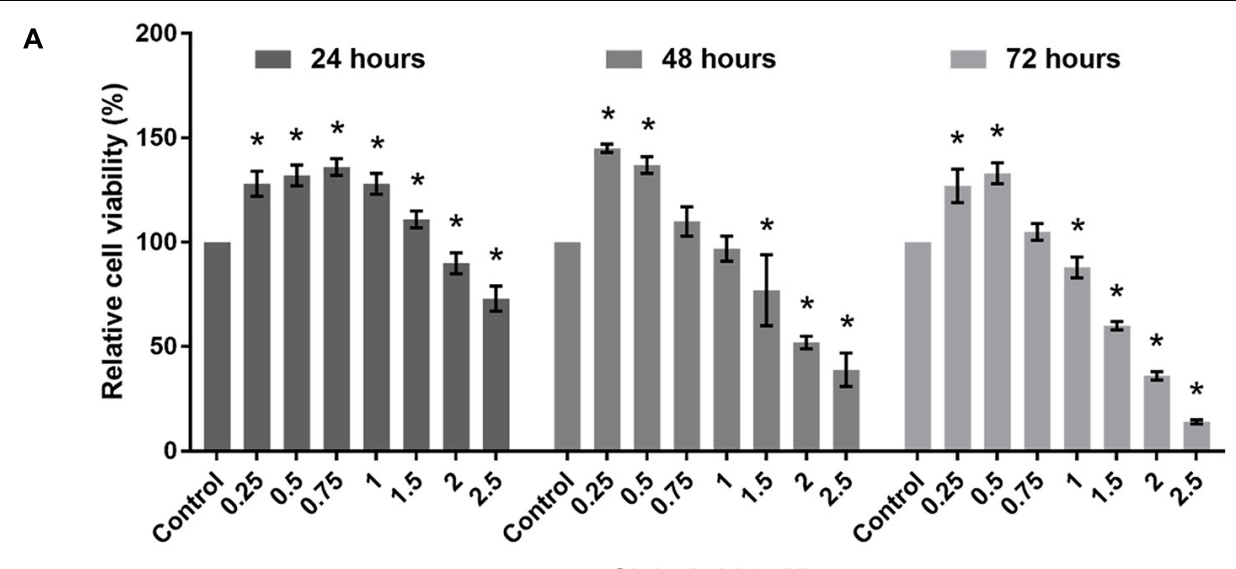

Oleic Acid (mM)

B
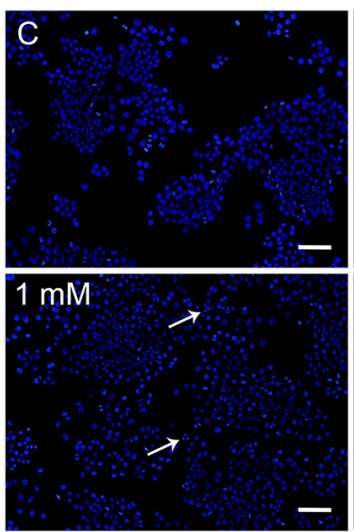
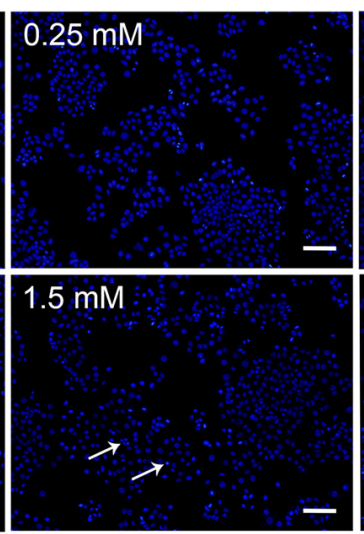
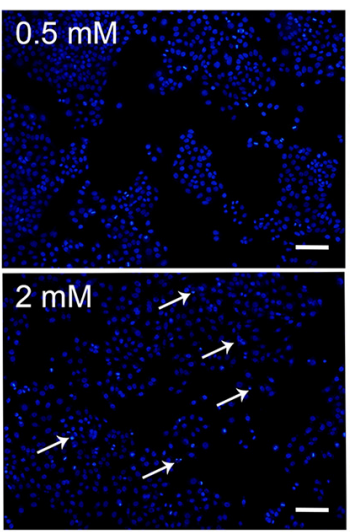
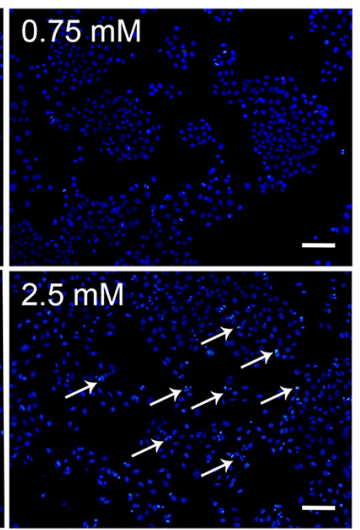

FIGURE 2 | Toxicity of OA on HepG2 cells. (A) Proliferation ratio of HepG2 cells treated with different concentrations of OA for 24, 48 , and 72 h. (B) The evaluation of apoptosis for $24 \mathrm{~h}$. Hoechst 33342 was used to stain the nuclei. Apoptotic cells were indicated by white arrowheads. Images taken at $10 \times$ magnification. Scale bars: $100 \mu \mathrm{m}$. Experiments were performed in triplicates in three independent experiments. ${ }^{*} p<0.05$, compared with the control groups.

to $31.17 \% \pm 1.75 \%$ (Figure $3 \mathrm{~B}$ ). The extent of ORO staining quantified by spectrophotometry also confirmed that OA could induce different proportions of intracellular lipid deposition (Figure 3C). The amount of ORO extracted in the 2 and $2.5 \mathrm{mM}$ groups was reduced compared with the $1.5 \mathrm{mM}$ group, and this phenomenon was related to the decrease in the total cell number. The absorbance was significantly higher than the control group $(p<0.05)$. Therefore, the in vitro model of lipid accumulation established by OA can simulate the main characteristics of human fatty liver in a dose-dependent manner for further analysis.

\section{Effects of OA on Actin Cytoskeleton Arrangement}

The actin cytoskeleton arrangement was observed by phalloidin staining (Figure 4). Each group showed parallel bundles of stress fiber in HepG2 cells (Figure 4A). With the increase in concentration, the directional arrangement of the stress fiber remained. The density of actin bundles decreased in the $1.5 \mathrm{mM}$ group, and the nuclei appear as chromatin condensation, suggesting the occurrence of apoptosis. The analysis of fluorescence also showed a weaker intensity in the
$1.5 \mathrm{mM}$ group $(p<0.05$; Figure $4 \mathrm{~B})$. Therefore, the actin filaments may not show a marked change within a certain concentration of lipid deposition.

\section{Effect of OA on the Viscoelastic Response of HepG2 Cells}

Figure 5A shows the average indentation depth of HepG2 cells as a function of time after the given different concentrations of OA. Under the same experimental conditions, the creep compliance increased with OA concentration. The power law model was used to fit the experiment as previously described. Two parameters were obtained, namely, the effective pre-factor compliance $\left(1 / E_{0}\right)$ at time $\tau_{0}$, and the power law exponent $\beta$. The $1 / E_{0}$ and $\beta$-values showed an increased tendency with lipid accumulation (Figure 5B and Table 1).

\section{DISCUSSION}

Steatosis is recognized in multiple liver diseases and characterized by the deposition of fat droplets (Petäjä and Yki-Järvinen, 2016). The diagnosis for steatosis is important for the early prevention 
A


B
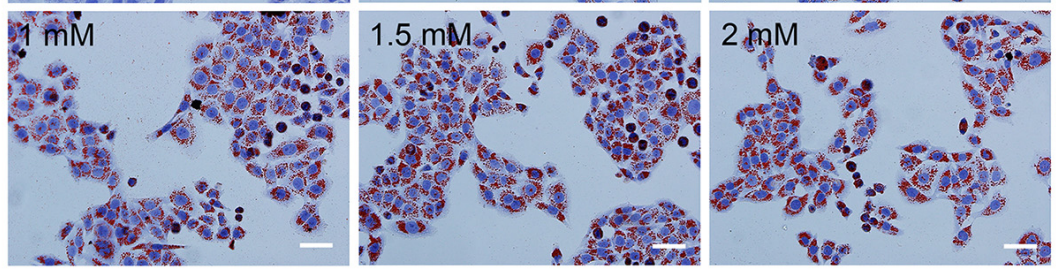

C

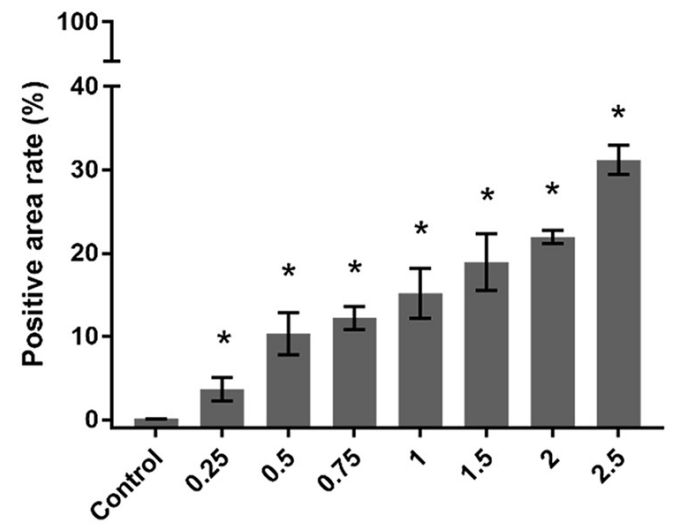

Oleic Acid (mM)

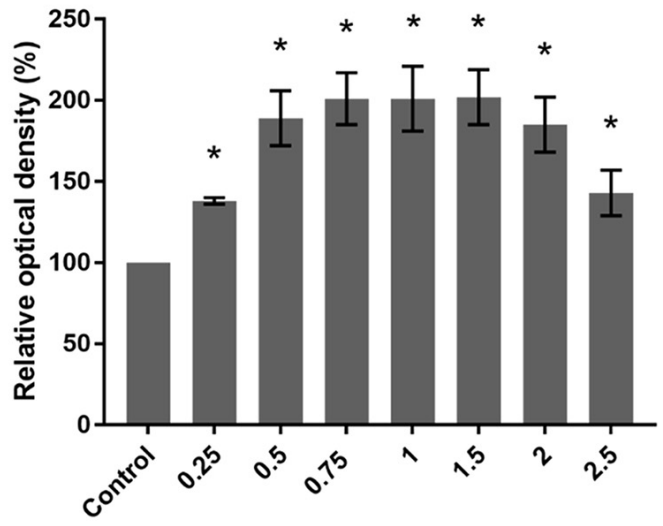

Oleic Acid (mM)

FIGURE 3 | Effects of OA concentration on lipid accumulation in HepG2 cells. (A) ORO staining results of HepG2 cells treated with different concentrations of OA for $24 \mathrm{~h}$. Red staining represents lipids. Images taken at $20 \times$ magnification. Scale bars: $50 \mu \mathrm{m}$. (B) Positive staining area of ORO staining. (C) Extent of ORO staining quantified by spectrophotometry. Experiments were performed in triplicates in three independent experiments. ${ }^{*} p<0.05$, compared with the control groups.

of various liver diseases (Diehl, 2010). In the present work, we used a hepatocyte deposition model with a gradient change to simulate intracellular lipid deposition. In comparison with other fatty acids such as palmitic acid, OA has higher lipid deposition effect and is less damaging (Ricchi et al., 2009). Therefore, OA was selected to amplify lipid deposition and reduce the apoptosis at a certain concentration. The semiquantitative analysis of ORO staining showed an increased positive area from 3.64 to $19 \%$. The AFM results showed that cell viscoelasticity is obviously concentration-related with lipid accumulation in cells, even when the lipid content is very low. Therefore, viscoelastic parameters can be used to classify light lipid deposition, providing a new insight into the classification of hepatic steatosis, especially in the early stage of the disease.

A significant amount of work has been done in noninvasive diagnostic methods in fatty liver. Yin et al. (2007) demonstrated that softer adipose tissue did not affect the overall stiffness of the liver. The stiffness range of fatty tissue is similar to the normal liver, indicating that the existence of hepatic steatosis in liver tissue does not directly affect the shear stiffness. Barry et al. (2014) found that fat can increase the viscosity of mouse livers, indicating that the viscosity may result in steatosis scoring. Zhu et al. (2015) used gelatinbased phantoms containing different ratios of castor oil to mimic different degrees of steatosis. Their results showed that Young's modulus decreases, whereas viscosity increases with increasing oil ratio (Zhu et al., 2015). The application of fluorescent probe to visualize intracellular viscosity also proved that fatty liver presents significant fluorescence compared with the healthy liver, indicating the high viscosity in fatty tissue (Yin et al., 2019). Although significant progresses have been made on qualitatively characterizing the mechanical properties changes in liver tissue during hepatic steatosis, both analysis methods and quantitative results are very limited for the accurate evaluation of the status of lipid deposition in single cell level. The present results on the changes of softness and fluidity of HepG2 cells clearly show the 




quantitative dependence on lipid deposition. This quantitative rule effectively eliminates the interference of the complexity and uncertainty of the mechanical microenvironment of liver tissue, accurately explains the quantitative relationship between the lipid deposition and the changes of observable mechanical indices during fatty liver disease progression, and provides valuable basis for the clinical diagnosis of this disease in the future.

In addition, as the primary structure to maintain the morphology of cells, studies have focused on the mechanical behavior caused by the remodeling of cytoskeleton (Satcher and Dewey, 1996; Rotsch and Radmacher, 2000; Pegoraro et al., 2017) and confirmed the major contribution of actin filament in cell elasticity (Kim et al., 2013; Grady et al., 2016; Sun et al., 2016). However, apart from cytoskeleton and binding proteins, subcellular organelles and crowding protein in cytoplasm may contribute to the rheology of the cells. These components are directly correlated with physiological and pathological process and hard to be solely defined in terms of elastic parameters. Our results showed that the effect of lipid accumulation on the actin might be limited in a certain degree, while mechanical response changes over time. According to soft glassy rheology theory, the degree of internal disorder in the cell can regulate its rheological behavior. Considering that the power-law constitutive relation is adopted, such a rheological behavior can be reflected by the powerlaw exponent, $\beta$, as shown in Equation (2) (Kollmannsberger and Fabry, 2011; Efremov et al., 2020). The cells behave more fluid-like when $\beta$ is close to 1 and more solid-like when $\beta$ approaches 0 (Kollmannsberger and Fabry, 2011). To investigate whether the rheological behavior changes with the intracellular environment, we altered the cellular components by adding lipids. Our results showed that the liver cells tend to be more fluid-like as the lipid concentration increases. The deposition of lipids occupied a limited intracellular space, thus possibly leading to aggravated intracellular crowding. This assumption 



FIGURE 5 | Cell viscoelastic feedback under different concentrations of OA in AFM creep measurements for 24 h. (A) Changes of indentation depth as functions of time after treatment of HepG2 cells with different concentrations of $\mathrm{OA}$. The circle marks represent the average of at least 60 indentation tests. Three independent experiments were performed. (B) Creep compliance response of HepG2 cells after dimensionless.

matches the experimental trends in which lipid accumulation increased with $\beta$.

Our phalloidin staining results revealed that F-actin remained intact within a certain degree of lipid deposition, whereas the values of $1 / E_{0}$ and $\beta$ increased with increased lipid deposition. Therefore, the contribution of actin cytoskeleton to the increased $1 / E_{0}$ might be limited in our study. The $1 / E_{0}$ value increased when the cytoskeleton remained unchanged possibly because of the proportion competition between the "hard-phase" (cytoskeleton) and the "soft-phase" (lipid) in the cells. As the lipid deposition increases, the "soft-phase" becomes increasingly dominant, resulting in the decrease in stiffness and increase in $1 / E_{0}$. This finding explains the observed $1 / E_{0}$ increase with the OA concentration in a dose-dependent manner. Interestingly, these changes in mechanical properties were mostly caused by the intracellular lipid deposition, rather than the cytoskeleton remodeling, thus providing a new insight into the understanding of the viscoelastic properties of liver cells.

In summary, we discussed the possibility of using viscoelastic feature of single cells to describe the hepatic steatosis. The results showed that the degree of lipid deposition in liver cells was

TABLE 1 | Least-square fitting parameters for the power-law model.

\begin{tabular}{|c|c|c|c|}
\hline Concentration (mM) & $1 / E_{0}\left(\mathrm{~Pa}^{-1}\right)$ & $\tau_{0}(\mathrm{~s})$ & $\beta$ \\
\hline 0 & 1.49E-03 & 7.53E-05 & 0.116 \\
\hline 0.25 & $1.84 \mathrm{E}-03$ & 7.04E-05 & 0.121 \\
\hline 0.5 & $2.14 \mathrm{E}-03$ & $6.63 E-05$ & 0.126 \\
\hline 0.75 & 2.26E-03 & 6.33E-05 & 0.127 \\
\hline 1 & 2.44E-03 & 6.29E-05 & 0.130 \\
\hline 1.5 & 2.45E-03 & 5.96E-05 & 0.132 \\
\hline
\end{tabular}

quantitatively correlated with elastic compliance and power-law parameters of the viscoelasticity. The viscoelastic property change in single cells may provide an important potential choice for early hepatic steatosis grading, and the viscoelasticity index might be an accurate detection indicator.

\section{DATA AVAILABILITY STATEMENT}

The raw data supporting the conclusions of this article will be made available by the authors, without undue reservation.

\section{AUTHOR CONTRIBUTIONS}

JW, RL, and YB conceived the design and wrote the manuscript. JW supervised the study and reviewed the manuscript. RL performed most experiments. RL, YB, and CY analyzed the data. JW and YB contributed to the theoretical analysis. All authors contributed to the article and approved the submitted version.

\section{FUNDING}

This work was supported by the National Natural Science Foundation of China (Grant No. 11925204).

\section{ACKNOWLEDGMENTS}

We thank the Experimental Center of Stomatology (Lanzhou University). 


\section{REFERENCES}

Barry, C. T., Hah, Z., Partin, A., Mooney, R. A., Chuang, K. H., Augustine, A., et al. (2014). Mouse liver dispersion for the diagnosis of early-stage fatty liver disease: a 70-sample study. Ultrasound Med. Biol. 40, 704-713. doi: 10.1016/j. ultrasmedbio.2013.10.016

Braet, F., Taatjes, D. J., and Wisse, E. (2018). Probing the unseen structure and function of liver cells through atomic force microscopy. Semin. Cell. Dev. Biol. 73, 13-30. doi: 10.1016/j.semcdb.2017.07.001

Bu, Y., Li, L., Yang, C., Li, R., and Wang, J. (2019). Measuring viscoelastic properties of living cells. Acta. Mech. Solida. Sin. 32, 599-610. doi: 10.1007/s10338-01900113-7

Castera, L., Friedrich-Rust, M., and Loomba, R. (2019). Noninvasive assessment of liver disease in patients with nonalcoholic fatty liver disease. Gastroenterology 156, 1264-1281.e1264. doi: 10.1053/j.gastro.2018.12.036

Costa, K. D. (2003). Single-cell elastography: probing for disease with the atomic force microscope. Dis. Markers. 19, 139-154. doi: 10.1155/2004/482680

Cousin, S. P., Hügl, S. R., Wrede, C. E., Kajio, H., Myers, M. G. Jr., and Rhodes, C. J. (2001). Free fatty acid-induced inhibition of glucose and insulin-like growth factor I-induced deoxyribonucleic acid synthesis in the pancreatic beta-cell line INS-1. Endocrinology 142, 229-240. doi: 10.1210/endo.142.1.7863

Cui, W., Chen, S. L., and Hu, K. Q. (2010). Quantification and mechanisms of oleic acid-induced steatosis in HepG2 cells. Am. J. Transl. Res. 2, 95-104.

Dasarathy, S., Dasarathy, J., Khiyami, A., Joseph, R., Lopez, R., and McCullough, A. J. (2009). Validity of real time ultrasound in the diagnosis of hepatic steatosis: a prospective study. J. Hepatol. 51, 1061-1067. doi: 10.1016/j.jhep.2009.09.001

Deffieux, T., Gennisson, J. L., Bousquet, L., Corouge, M., Cosconea, S., Amroun, D., et al. (2015). Investigating liver stiffness and viscosity for fibrosis, steatosis and activity staging using shear wave elastography. J. Hepatol. 62, 317-324. doi: 10.1016/j.jhep.2014.09.020

Desprat, N., Richert, A., Simeon, J., and Asnacios, A. (2005). Creep function of a single living cell. Biophys. J. 88, 2224-2233. doi: 10.1529/biophysj.104.050278

Diehl, A. M. (2010). Genetic susceptibility to hepatic steatosis. N. Engl. J. Med. 362, 1142-1143. doi: 10.1056/NEJMe1000206

Efremov, Y. M., Okajima, T., and Raman, A. (2020). Measuring viscoelasticity of soft biological samples using atomic force microscopy. Soft. Matter. 16, 64-81. doi: $10.1039 / \mathrm{c} 9 \mathrm{sm} 01020 \mathrm{c}$

Grady, M. E., Composto, R. J., and Eckmann, D. M. (2016). Cell elasticity with altered cytoskeletal architectures across multiple cell types. J. Mech. Behav. Biomed. Mater. 61, 197-207. doi: 10.1016/j.jmbbm.2016.01.022

Gu, J., Liu, S., Du, S., Zhang, Q., Xiao, J., Dong, Q., et al. (2019). Diagnostic value of MRI-PDFF for hepatic steatosis in patients with non-alcoholic fatty liver disease: a meta-analysis. Eur. Radiol. 29, 3564-3573. doi: 10.1007/s00330-01906072-4

Hernaez, R., Lazo, M., Bonekamp, S., Kamel, I., Brancati, F. L., Guallar, E., et al. (2011). Diagnostic accuracy and reliability of ultrasonography for the detection of fatty liver: a meta-analysis. Hepatology 54, 1082-1090. doi: 10.1002/hep. 24452

Hoyles, L., Fernández-Real, J.-M., Federici, M., Serino, M., Abbott, J., Charpentier, J., et al. (2018). Molecular phenomics and metagenomics of hepatic steatosis in non-diabetic obese women. Nat. Med. 24, 1070-1080. doi: 10.1038/s41591-0180061-3

Jiang, J., Jin, H., Liu, L., Pi, J., Yang, F., and Cai, J. (2013). Curcumin disturbed cell-cycle distribution of HepG2 cells via cytoskeletal arrangement. Scanning 35, 253-260. doi: 10.1002/sca.21058

Karlas, T., and Mueller, S. (2020). "Liver steatosis (CAP) as modifier of liver stiffness," in Liver Elastography: Clinical Use and Interpretation, ed. S. Mueller (Cham: Springer International Publishing), 459-467.

Kim, Y., Hong, J. W., Kim, J., and Shin, J. H. (2013). Comparative study on the differential mechanical properties of human liver cancer and normal cells. Anim. Cells Syst. 17, 170-178. doi: 10.1080/19768354.2013.789452

Knowles, B. B., Howe, C. C., and Aden, D. P. (1980). Human hepatocellular carcinoma cell lines secrete the major plasma proteins and hepatitis B surface antigen. Science 209, 497-499. doi: 10.1126/science.6248960

Kollmannsberger, P., and Fabry, B. (2011). Linear and nonlinear rheology of living cells. Annu. Rev. Mater. Res. 41, 75-97. doi: 10.1146/annurev-matsci-062910100351
Krawitz, S., and Pyrsopoulos, N. (2020). "Nonalcoholic fatty liver disease (NAFLD) and nonalcoholic steatohepatitis (NASH)," in Geriatric Gastroenterology, eds C. Pitchumoni and T. Dharmarajan (New York, NY: Springer), 1-10.

Lee, S. S., and Park, S. H. (2014). Radiologic evaluation of nonalcoholic fatty liver disease. World J. Gastroenterol. 20, 7392-7402. doi: 10.3748/wjg.v20. i23.7392

Lee, S. S., Park, S. H., Kim, H. J., Kim, S. Y., Kim, M.-Y., Kim, D. Y., et al. (2010). Non-invasive assessment of hepatic steatosis: prospective comparison of the accuracy of imaging examinations. J. Hepatol. 52, 579-585. doi: 10.1016/j.jhep. 2010.01.008

Mazhar, S. M., Shiehmorteza, M., and Sirlin, C. B. (2009). Noninvasive assessment of hepatic steatosis. Clin. Gastroenterol. Hepatol. 7, 135-140. doi: 10.1016/j.cgh. 2008.11.023

Mueller, S. (2016). Does pressure cause liver cirrhosis? The sinusoidal pressure hypothesis. World J. Gastroenterol. 22, 10482-10501. doi: 10.3748/wjg.v22.i48. 10482

Mueller, S. (2020a). "Introduction to liver stiffness: a novel parameter for the diagnosis of liver disease," in Liver Elastography: Clinical Use and Interpretation, ed. S. Mueller (Cham: Springer International Publishing), 3-9.

Mueller, S. (2020b). "Liver Stiffness and Its Measurement," in Liver Elastography: Clinical Use and Interpretation, ed. S. Mueller (Cham: Springer International Publishing), 13-28.

Mueller, S., and Sandrin, L. (2010). Liver stiffness: a novel parameter for the diagnosis of liver disease. Hepat. Med. 2, 49-67. doi: 10.2147/hmer.s7394

Mueller, S., Elshaarawy, O., and Piecha, F. (2020). "Future applications and directions of liver stiffness studies," in Liver Elastography: Clinical Use and Interpretation, ed. S. Mueller (Cham: Springer International Publishing), 687-693.

Pegoraro, A. F., Janmey, P., and Weitz, D. A. (2017). Mechanical properties of the cytoskeleton and cells. cold spring harb. Perspect. Biol. 9:a022038. doi: 10.1101/cshperspect.a022038

Petäjä, E. M., and Yki-Järvinen, H. (2016). Definitions of normal liver fat and the association of insulin sensitivity with acquired and genetic NAFLD-a systematic review. Int. J. Mol. Sci. 17:633. doi: 10.3390/ijms1705 0633

Rausch, V., Peccerella, T., Lackner, C., Yagmur, E., Seitz, H.-K., Longerich, T., et al. (2016). Primary liver injury and delayed resolution of liver stiffness after alcohol detoxification in heavy drinkers with the PNPLA3 variant I148M. World J. Hepatol. 8, 1547-1556. doi: 10.4254/wjh.v8.i35.1547

Ricchi, M., Odoardi, M. R., Carulli, L., Anzivino, C., Ballestri, S., Pinetti, A., et al. (2009). Differential effect of oleic and palmitic acid on lipid accumulation and apoptosis in cultured hepatocytes. J. Gastroenterol. Hepatol. 24, 830-840. doi: 10.1111/j.1440-1746.2008.05733.x

Righetti, R., Ophir, J., and Ktonas, P. (2002). Axial resolution in elastography. Ultrasound Med. Biol. 28, 101-113. doi: 10.1016/S0301-5629(01) 00495-1

Righetti, R., Srinivasan, S., and Ophir, J. (2003). Lateral resolution in elastography. Ultrasound Med. Biol. 29, 695-704. doi: 10.1016/S0301-5629(03) 00028-0

Rockey, D. C., Caldwell, S. H., Goodman, Z. D., Nelson, R. C., and Smith, A. D. (2009). Liver biopsy. Hepatology 49, 1017-1044. doi: 10.1002/hep. 22742

Rotsch, C., and Radmacher, M. (2000). Drug-induced changes of cytoskeletal structure and mechanics in fibroblasts: an atomic force microscopy study. Biophys. J. 78, 520-535. doi: 10.1016/s0006-3495(00)76614-8

Saadeh, S., Younossi, Z. M., Remer, E. M., Gramlich, T., Ong, J. P., Hurley, M., et al. (2002). The utility of radiological imaging in nonalcoholic fatty liver disease. Gastroenterology 123, 745-750. doi: 10.1053/gast.2002.35354

Sanyal, A. J., Chalasani, N., Kowdley, K. V., McCullough, A., Diehl, A. M., Bass, N. M., et al. (2010). Pioglitazone, vitamin E, or placebo for nonalcoholic steatohepatitis. N. Engl. J. Med. 362, 1675-1685. doi: 10.1056/NEJMoa09 07929

Satcher, R. L. Jr., and Dewey, C. F. Jr. (1996). Theoretical estimates of mechanical properties of the endothelial cell cytoskeleton. Biophys. J. 71, 109-118. doi: 10.1016/s0006-3495(96)79206-8

Seitz, H. K., Bataller, R., Cortez-Pinto, H., Gao, B., Gual, A., Lackner, C., et al. (2018). Alcoholic liver disease. Nat. Rev. Dis. Primers 4:16. doi: 10.1038/s41572018-0014-7 
Sneddon, I. N. (1965). The relation between load and penetration in the axisymmetric boussinesq problem for a punch of arbitrary profile. Int. J. Eng. Sci. 3, 47-57. doi: 10.1016/0020-7225(65)90019-4

Sun, J., Luo, Q., Liu, L., Zhang, B., Shi, Y., Ju, Y., et al. (2016). Biomechanical profile of cancer stem-like cells derived from MHCC97H cell lines. J. Biomech. 49, 45-52. doi: 10.1016/j.jbiomech.2015.11.007

Tian, M., Li, Y., Liu, W., Jin, L., Jiang, X., Wang, X., et al. (2015). The nanomechanical signature of liver cancer tissues and its molecular origin. Nanoscale 7, 12998-13010. doi: 10.1039/c5nr02192h

Tsikritsis, D., Shi, H., Wang, Y., Velugotla, S., Sršeň, V., Elfick, A., et al. (2016). Label-free biomarkers of human embryonic stem cell differentiation to hepatocytes. Cytometry. A. 89, 575-584. doi: 10.1002/cyto.a.22875

Yang, W. H. (1966). The contact problem for viscoelastic bodies. J. Appl. Mech. 33, 395-401. doi: 10.1115/1.3625055

Yin, J., Peng, M., and Lin, W. (2019). Visualization of mitochondrial viscosity in inflammation, fatty liver, and cancer living mice by a robust fluorescent probe. Anal. Chem. 91, 8415-8421. doi: 10.1021/acs.analchem.9b01293

Yin, M., Talwalkar, J. A., Glaser, K. J., Manduca, A., Grimm, R. C., Rossman, P. J., et al. (2007). Assessment of hepatic fibrosis with magnetic resonance elastography. Clin. Gastroenterol. Hepatol. 5, 1207-1213.e1202. doi: 10.1016/j. cgh.2007.06.012

Yun, M. R., Lee, J. Y., Park, H. S., Heo, H. J., Park, J. Y., Bae, S. S., et al. (2006). Oleic acid enhances vascular smooth muscle cell proliferation via phosphatidylinositol 3-kinase/Akt signaling pathway. Pharmacol. Res. 54, 97102. doi: $10.1016 /$ j.phrs.2006.03.001
Zhang, B., Ding, F., Chen, T., Xia, L.-H., Qian, J., and Lv, G.-Y. (2014). Ultrasound hepatic/renal ratio and hepatic attenuation rate for quantifying liver fat content. World J. Gastroenterol. 20, 17985-17992. doi: 10.3748/wjg.v20.i47. 17985

Zhu, Y., Dong, C., Yin, Y., Chen, X., Guo, Y., Zheng, Y., et al. (2015). The role of viscosity estimation for oil-in-gelatin phantom in shear wave based ultrasound elastography. Ultrasound Med. Biol. 41, 601-609. doi: 10.1016/j.ultrasmedbio. 2014.09.028

Conflict of Interest: The authors declare that the research was conducted in the absence of any commercial or financial relationships that could be construed as a potential conflict of interest.

Publisher's Note: All claims expressed in this article are solely those of the authors and do not necessarily represent those of their affiliated organizations, or those of the publisher, the editors and the reviewers. Any product that may be evaluated in this article, or claim that may be made by its manufacturer, is not guaranteed or endorsed by the publisher.

Copyright (c) $2021 \mathrm{Li}, \mathrm{Bu}$, Yang and Wang. This is an open-access article distributed under the terms of the Creative Commons Attribution License (CC BY). The use, distribution or reproduction in other forums is permitted, provided the original author(s) and the copyright owner(s) are credited and that the original publication in this journal is cited, in accordance with accepted academic practice. No use, distribution or reproduction is permitted which does not comply with these terms. 